\title{
Informations SPE
}

\section{SPE news}

17 novembre 2010, Paris, France.

Réunion thématique de la Société de pathologie exotique : HTLV1, don de sang, don d'organes et assistance médicale à la procréation

\section{Programme provisoire}

14 h 00 : Historique, épidémiologie clinique et moléculaire, aspects cliniques principaux. Antoine Gessain, Institut Pasteur 14 h 30 : Aspects virologiques d'HTLV1. Renaud Mahieux, École normale de Lyon

14 h 45 : Transfusion sanguine

- Méthodes de prévention de la transmission de l'HTLV chez les donneurs de sang en France et à l'étranger. Azzedine Assal, Établissement français du sang

- Épidémiologie de l'HTLV chez les donneurs de sang depuis mi-1991 à 2008. Josiane Pillonel, Institut national de veille sanitaire

15 h 15 : Assistance médicale à la procréation

- AMP en contexte viral HTLV1. Emmanuel Dulioust, CHU Cochin et Guillaume Joguet, CHU de Pointe-à-Pitre

- Dépistage ciblé HTLV1 des couples demandant une AMP. Sylvie Epelboin, CHU Bichat

- HTLV1 en AMP. Christophe Pasquier, Institut fédératif de biologie, Toulouse

16 h 00 : Greffes

Les limites du principe de précaution : bénéfice/risque du dépistage d'HTLV1 avant prélèvement d'organes et de tissus. Corinne Antoine, CHU Saint-Louis

16 h 35 : Varia

Anguillules et HTLV1. Jean-François Pays

Information : SPE, 20, rue Ernest-Renan, F-75015 Paris, France

Courriel : socpatex@pasteur.fr

\section{Informations générales}

\section{8 au 30 juin 2011, Lyon, France. \\ Premier Congrès international francophone d'accompagnement et de soins palliatifs}

La Société française d'accompagnement et de soins palliatifs (SFAP) organise à Lyon, du 28 au 30 juin 2011, dans le contexte de son $17^{\mathrm{e}}$ congrès national, le premier Congrès international francophone d'accompagnement et de soins palliatifs.

Le thème choisi pour ce congrès est : «À la rencontre de nos diversités ».
Cinq thématiques transversales ont été retenues :

- la douleur ;

- l'accompagnement de la personne malade et de ses proches ;

- l'histoire des pratiques de soins, des législations et des politiques de santé en soins palliatifs ;

- l'agonie et le moment de la mort ;

- la mort provoquée, la sédation, la limitation thérapeutique. Outre les communications en séance plénière, chacune de ces thématiques transversales sera déclinée sous forme d'ateliers :

- cours de sensibilisation ou «bases essentielles » en soins palliatifs ;

- cours de perfectionnement;

- sujets de controverses, de débat ;

- présentation d'innovations, d'approches singulières ;

- présentation de travaux en lien avec la recherche (études terminées ou non, projets, réflexion sur les méthodes, etc.).

D'autres thématiques feront l'objet de communications en ateliers :

- soins palliatifs et gériatrie ;

- soins palliatifs et pédiatrie ;

- soins palliatifs et bénévolat ;

- soins palliatifs et pathologies spécifiques (sida, SLA, etc.);

- démarche palliative selon le lieu de prise en charge : dans les services d'urgences et de réanimation, à domicile, etc. ;

- autres symptômes que la douleur (agitation, confusion, fatigue, cachexie, etc.) ;

- soins du corps (approches innovantes, place de la famille, place de la culture, etc.) ;

- rites funéraires ;

- deuil ;

- alimentation et culture ;

- souffrance des soignants (prévention, repérage, accompagnement, etc.) ;

- enseignement, méthodes pédagogiques et recherche ;

- processus décisionnels (les différentes étapes qui conduisent à la décision, les positionnements de chacun des acteurs, les repères éthiques, législatifs, etc.) ;

- le travail en équipe.

Renseignements et inscriptions sur le site des congrès de la SFAP : http://congres.sfap.org/.

La date limite pour le dépôt des propositions d'intervention avec résumés est fixée au 25 octobre 2010. Modalités de soumission sur le site http://congres.sfap.org/.

Président du comité scientifique international : Dr Vincent Morel, médecin, CHU de Rennes (France) : vincent.morel@ chu-rennes.fr 